\title{
Knowledge of sex partner treatment for past bacterial STI and risk of current STI
}

\author{
L M Niccolai, J R Ickovics, K Zeller, T S Kershaw, S Milan, J B Lewis, K A Ethier
}

Sex Transm Infect 2005;81:271-275. doi: 10.1136/sti.2004.012872

See end of article for authors' affiliations

........................

Correspondence to: Linda M Niccolai, PhD, Yale University School of Medicine, Department of Epidemiology and Public Health, 60 College Street, PO Box 208034, New Haven, CT 06520-8034, USA; linda.niccolai@ yale.edu

Accepted for publication 21 September 2004

\begin{abstract}
Objectives: Effective partner management is critical in reducing the spread of bacterial sexually transmitted infections (STIs). The purpose of this study was to determine the relation between knowledge of partner treatment for a past STI and current infection in the index patient.

Methods: In a cross sectional analysis, 97 adolescent females sampled from community based health clinics reported that they had a past diagnosis of chlamydia or gonorrhoea in structured, face to face interviews. At the time of the interview, adolescents were also tested for chlamydia and gonorrhoea using urine based ligase chain reaction testing.

Results: $66 \%$ of the adolescents reported knowing that their partner was treated for the past infection. Those who knew their partner was treated were less likely to have a current infection, compared to those who did not know (11\% v 30\%, adjusted odds ratio and 95\% confidence interval 4.46 (1.41 to 14.29), $\mathrm{p}<0.05)$. Correlates of not knowing the sex partner was treated included younger age and being in new sex partnership.

Conclusions: Efforts to encourage young women to follow up directly with their partners regarding treatment may help to reduce repeat infections and further spread. Furthermore, alternative strategies such as patient delivered therapy may help with partner treatment in this vulnerable population.
\end{abstract}


hlamydia (caused by Chlamydia trachomatis) and gonorrhoea (caused by Neisseria gonorrhoeae) are common among young females, and many adolescents are likely to have repeat infections. ${ }^{1-8}$ These repeat infections may represent a new infection, re-infection from a previously untreated or non-monogamous partner, treatment failure, or persistence. Repeat infections can have major negative health consequences, including pelvic inflammatory disease, chronic pelvic pain, pregnancy complications, and infertility. ${ }^{10}$ Furthermore, these bacterial sexually transmitted infections (STI) increase the risk of HIV transmission. ${ }^{11}{ }^{12}$

Partner treatment is an essential component of preventing re-infection in the index patient. ${ }^{13}$ Partner management strategies may include patient referral, in which the index patient tells the partner directly to seek treatment; healthcare provider referral, in which clinicians contact the partner using locating information provided by the patient; or partner notification through the public health system, in which community health workers locate partners in the community. Furthermore, more innovative methods are gaining widespread recognition, including patient delivered therapy, in which patients are provided with medications to take to partners ${ }^{14-16}$; field delivered therapy, in which trained healthcare workers take medication to partners who do not come into the $\operatorname{clinic}^{17}$; and expedited partner treatment through commercial pharmacies. ${ }^{18}$ While all of these methods have met with important success, their utilisation is limited. Results from a national survey indicated that only $4-7 \%$ of largely private practice clinicians reported always conducting partner notification for their STI patients. ${ }^{19}$ In another survey, only $17 \%$ of private sector clinicians knew that all of their patients' partners were treated..$^{20}$ Furthermore, a recent study showed that partner notification services were provided to only a very small number of patients with chlamydia and gonorrhoea in public health departments ( $12 \%$ and $17 \%$, respectively). ${ }^{21}$

These studies suggest that patients themselves can and should play an important part in the treatment of their partners. In order for patient based efforts to be successful, individuals must tell their partners about the infection, provide assistance to their partners in obtaining services, and ensure that all partners were treated. Although the first of these steps has been the focus of some attention, the last and very important step has not. If women continue to engage in sex with partners while not knowing whether their partners were treated, their risk for repeat infections is greatly increased.

The purpose of this study was to examine the relation between patients' knowledge of whether their partners were treated for a past chlamydia or gonorrhoea diagnosis and risk of current infections in the index patients, and to determine correlates of knowledge of partner treatment.

\section{METHODS \\ Sample}

This study utilised data from a research project examining risks for HIV/STIs among adolescent females, a population with high STI prevalence, conducted from 1998 to 2000. Participants were recruited from public health clinics that provide reproductive health services. Eligibility criteria for participation in the larger study included being a female between the ages of 14 and 19, ever having had sexual intercourse, never having given birth, and being HIV negative. Per study protocol, half of the participants were pregnant at enrolment. Participants were recruited by referral from clinicians, responding to advertising materials in the clinics, study staff approaching patients in the waiting rooms, or word of mouth.

\section{Data collection}

Participants completed structured, face to face interviews that ascertained demographic information, sexual histories, current sexual risk behaviours, partnership characteristics, and STI history. Among those who reported a diagnosis of chlamydia or gonorrhoea, the following questions were also asked: "The last time you were diagnosed with any of these

Abbreviations: LCR, ligase chain reaction; STI, sexually transmitted infections 
sexually transmitted infections, did your healthcare provider recommend that your boyfriend or husband get tested?", "Did you tell your boyfriend or husband the last time you were diagnosed with any of these sexually transmitted infections?", and "Did your boyfriend or husband get treated by a healthcare provider the last time you were diagnosed with any of these sexually transmitted infections?" Knowledge of partner treatment was determined by patient's response to the last question. Responses of "yes" were coded as knowledge that the partner was treated, and responses of "no" or "don't know" were coded as not having knowledge that the partner was treated. This categorisation separated those who knew their partners were treated by presumably taking some steps to ensure this from those who lacked knowledge that the partner was treated, and is similar to the grouping used in previous research. ${ }^{5}$

Covariates of interest included demographic, sexual risk, and partnership variables. Demographic factors included age (14-16 v 17-19), race (African-American, Hispanic, white/ other), and education (high school diploma or in school $v$ no high school diploma and not in school). Sexual risk factors included ever pregnant (yes $v$ no), age at first intercourse $(\leqslant 14 v>14)$, number of sex partners per year of sexual activity $(\leqslant 1 \quad v>1)$, unprotected intercourse in the past 30 days (yes $v$ no), alcohol or drug use before sex in past 30 days (yes $v$ no), and current number of sex partners defined as the number in the past 30 days $(\leqslant 1 v>1)$. We chose to examine number of sex partners per year of sexual activity because this is a sample of adolescents aged 14-19 in which there are great variations in the durations of their sexually active lifetimes. Thus, a measure of total number of lifetime sex partners may not be directly comparable. The partnership variables included relationship duration $(\leqslant 1 \mathrm{v}$ $>$ l year), partner risk history (yes $v$ no, partner risks included history of injection drug use, history of STI, or sex with other partners in past 6 months), and whether the participant was in the same partnership at the time of the reported past diagnosis and the time of the interview and STI testing for current infections.

Testing for current chlamydia and gonorrhoea infections was conducted using ligase chain reaction (LCR) testing (Abbott Laboratories, Chicago, IL, USA). Urine samples were collected during the study visit and LCR testing was conducted at a central laboratory. Participants were classified as having a current chlamydia or gonorrhoea infection if the urine sample collected at the time of the interview tested positive for the same infection (chlamydia or gonorrhoea) reported in the past. Institutional review boards at all participating sites and institutions approved this study for its ethical conduct. Participation was completely voluntary and confidential, and study participants gave written informed consent to participate in this study. Participants were paid $\$ 25$ for the interview.

\section{Analysis}

Statistical analyses were restricted to the subgroup of participants who reported a past diagnosis of chlamydia or gonorrhoea. Likelihood ratio $\chi^{2}$ tests and multivariate logistic regression were used to determine the association between knowledge of partner treatment for past diagnosis and current infection. Knowledge of partner treatment, the main predictor of interest, was included in all models. Covariates that were associated in the bivariate analysis using the critical value of $p<0.20$ were included in the initial multivariate model. A backward selection procedure was used to eliminate covariates that did not remain significant using the critical value of $\mathrm{p}<0.05$ or confound the main relation of interest. Correlates of knowledge of partner treatment were determined using likelihood ratio $\chi^{2}$ tests.

\section{RESULTS}

A total of 411 (77\% of eligible screened) adolescent females were enrolled in the study; 104 reported a past diagnosis of chlamydia or gonorrhoea. Of these 104, the 97 who had valid responses on the main variables of interest were included in the present analyses. Self reports of past diagnoses were compared to two external data sources that were available for this sample: medical record reviews and state health department reports. Among those in the entire sample who had these external sources available $(n=361,89 \%)$, the prevalence of past diagnoses by self report, medical record reviews, and state health department reports were not significantly different for chlamydia $(20.5 \%, 23.0 \%, 19.7 \%$, respectively) or gonorrhoea $(4.7 \%, 6.9 \%$, and $5.5 \%$, respectively) ( $\mathrm{p}>.05$ for all comparisons).

Sample characteristics are described in table 1. The average age was 17.3 (SD 1.5) years. The average age at first intercourse was 14.5 (1.6) years and many had unprotected intercourse in the past 30 days $(51 \%)$. Current pregnancy status was not associated with knowledge of partner treatment or current infection, the main variables of interest, so these two groups were combined for all analyses. The majority of participants (94\%) reported being in a monogamous partnership. The median duration of the current sexual partnerships among those reporting a current partner $(\mathrm{n}=83)$ was 12.5 months (range 0.1-92.8 months).

The majority of self reported past STIs were chlamydia ( $\mathrm{n}=88,91 \%)$ with a smaller number of gonorrhoea infections $(\mathrm{n}=21,22 \%)$; a small proportion $(\mathrm{n}=12,12 \%)$ reported both. Medical record data with STI diagnosis information were available for 74 (76\%) participants; these records confirmed treatment for 69 of those women (93\%). The median time interval from the reported date of the past diagnosis to the date of the interview was 5.9 months (range 0.3-74.9 months). Ninety five per cent $(n=92)$ reported that their healthcare provider recommended partner treatment and $81 \%(n=79)$ reported that they told their partners about the infection. Sixty six per cent $(n=64)$ reported knowing that their partners were treated for the past infection ( $81 \%$ of those who reported telling her partner about the infection), and $34 \%(n=33)$ reported not knowing that their partners were treated, including $26 \%(\mathrm{n}=25)$ who said their partners were not treated and $8 \%(\mathrm{n}=8)$ who reported not knowing.

Seventeen participants ( $18 \%$ ) had a current chlamydia or gonorrhoea infection at the time of the interview (16 chlamydia, three gonorrhoea (two had both)). Among those who knew their partner was treated for the past infection, $11 \%(7 / 64)$ had a current infection, compared to $30 \%(10 / 33)$ of those who did not know their partner was treated $(p=0.02)$. The likelihood of current infection was not significantly different between those who reported their partner was not treated and those who reported they did not know if their partner was treated $(p=0.38)$. These two groups were combined for the remainder of analyses because of the small number who reported not knowing $(n=8)$. Unadjusted and adjusted odds ratios from the logistic regression model are presented in table 2 . In the final model, the odds of a current infection were 4.5 times greater $(95 \%$ CI: 1.4 to $14.3, p=0.01$ ) among those who did not know their partner was treated compared to those who did know their partner was treated. Excluding those who did not know if their partner was treated $(n=8)$ from the model in order to compare those who knew their partner was treated and those who reported knowing their partner was not treated resulted in similar results $(\mathrm{OR}=4.8,95 \% \mathrm{CI}: 1.5$ to $15.4, \mathrm{p}=0.01)$.

Post hoc analyses were conducted to explore the effect of having a different sex partner between the time of the self reported past diagnosis and the time of testing for current STI. The above logistic regression model was run on a 
Table 1 Infection prevalence with chlamydia or gonorrhoea among female adolescents with a past diagnosis of chlamydia or gonorrhoea by demographic and behavioural characteristics ( $n=97$ unless otherwise noted)

\begin{tabular}{|c|c|c|c|}
\hline & $\begin{array}{l}\text { Current infection } \\
(n=17)\end{array}$ & $\begin{array}{l}\text { No infection } \\
(n=80)\end{array}$ & p Value ${ }^{*}$ \\
\hline \multicolumn{4}{|l|}{ Age (years) } \\
\hline $14-16$ & $3(11 \%)$ & $24(89 \%)$ & \multirow[t]{2}{*}{0.24} \\
\hline $17-19$ & $14(20 \%)$ & $56(80 \%)$ & \\
\hline \multicolumn{4}{|l|}{ Race } \\
\hline African-American & $9(17 \%)$ & $44(83 \%)$ & \multirow{3}{*}{0.10} \\
\hline Hispanic & $8(23 \%)$ & $26(77 \%)$ & \\
\hline White/other & $0(05)$ & $10(100)$ & \\
\hline \multicolumn{4}{|l|}{ Educationt } \\
\hline In HS or HSD & $12(16 \%)$ & $65(84 \%)$ & \multirow{2}{*}{0.33} \\
\hline Not in HS and no HSD & $5(25 \%)$ & $15(75 \%)$ & \\
\hline \multicolumn{4}{|l|}{ Ever pregnant } \\
\hline Yes & $10(17 \%)$ & $48(83 \%)$ & \multirow[t]{2}{*}{0.99} \\
\hline No & $7(18 \%)$ & $32(82 \%)$ & \\
\hline \multicolumn{4}{|c|}{ Age at first intercourse (years) } \\
\hline$\leqslant 14$ & $8(15 \%)$ & $47(86 \%)$ & \multirow[t]{2}{*}{0.38} \\
\hline$>14$ & $9(21 \%)$ & $33(79 \%)$ & \\
\hline \multicolumn{4}{|c|}{ Number of partners per year of sexual activity } \\
\hline$\leqslant 1$ & $4(12 \%)$ & $30(88 \%)$ & \multirow[t]{2}{*}{0.26} \\
\hline$>1$ & $13(21 \%)$ & $50(79 \%)$ & \\
\hline \multicolumn{4}{|c|}{ Unprotected intercourse in the past 30 days } \\
\hline Yes & $13(27 \%)$ & $36(73 \%)$ & \multirow[t]{2}{*}{0.02} \\
\hline No & $4(8 \%)$ & $44(92 \%)$ & \\
\hline \multicolumn{4}{|c|}{ Alcohol or drug use before last sexual intercourse $(n=87)$} \\
\hline Yes & $3(14 \%)$ & $19(86 \%)$ & \multirow{2}{*}{0.32} \\
\hline No & $14(22 \%)$ & $51(78 \%)$ & \\
\hline \multicolumn{4}{|c|}{ Number of sex partners in past 30 days } \\
\hline$\leqslant 1$ & $15(17 \%)$ & $76(83 \%)$ & \multirow[t]{2}{*}{0.28} \\
\hline$>1$ & $2(33 \%)$ & $4(67 \%)$ & \\
\hline \multicolumn{4}{|c|}{ Current relationship duration in years $(n=82)$} \\
\hline$\leqslant 1$ & $11(29 \%)$ & $27(71 \%)$ & \multirow[t]{2}{*}{0.01} \\
\hline$>1$ & $3(7 \%)$ & 41 (93\%) & \\
\hline \multicolumn{4}{|l|}{ Partner riskł } \\
\hline Yes & $9(20 \%)$ & $37(80 \%)$ & \multirow[t]{2}{*}{0.62} \\
\hline No & $8(16 \%)$ & $43(84 \%)$ & \\
\hline \multicolumn{4}{|c|}{ Knowledge of partner treatment for past infection } \\
\hline No/don't know & $10(30 \%)$ & $23(70 \%)$ & \\
\hline
\end{tabular}

*By likelihood ratio $\chi^{2}$ test or Fisher's exact where appropriate. tHS, high school; HSD, high school diploma.

flncludes ever injection drug use, history of sexually transmitted infection, recent sex with others.

Table 2 Relation between partner treatment for past diagnosis and current infection $(n=97)$

\begin{tabular}{|c|c|c|}
\hline & $\begin{array}{l}\text { Unadjusted OR } \\
(95 \% \mathrm{Cl}) \S\end{array}$ & $\begin{array}{l}\text { Adjusted OR } \\
(95 \% \mathrm{Cl}) \S\end{array}$ \\
\hline \multicolumn{3}{|c|}{ Knowledge of partner treatment for past diagnosis $\dagger$} \\
\hline No $v$ yes & $\begin{array}{l}2.92 \\
(0.80 \text { to } 10.64)^{* *}\end{array}$ & $\begin{array}{l}4.46 \\
(1.41 \text { to } 14.29)^{*}\end{array}$ \\
\hline \multicolumn{3}{|l|}{ Race } \\
\hline Hispanic v African-American & $\begin{array}{l}1.39 \\
(0.41 \text { to } 4.76)\end{array}$ & NS \\
\hline \multicolumn{3}{|c|}{ Average number of partners per year of sexual activity } \\
\hline$>1 v \leqslant 1$ & $\begin{array}{l}1.23 \\
(0.31 \text { to } 4.94)\end{array}$ & NS \\
\hline \multicolumn{3}{|c|}{ Unprotected intercourse in past 30 days } \\
\hline Yes $v$ no & $\begin{array}{l}7.96 \\
(1.93 \text { to } 32.43)^{* * * *}\end{array}$ & $\begin{array}{l}4.98 \\
(1.40 \text { to } 17.77)^{*}\end{array}$ \\
\hline
\end{tabular}

${ }^{*} \mathrm{p}<0.05$.

tPartner treatment for past STI defined as no if the patient did not know if the partner was treated or if the patient thought the partner was not treated, and yes if the patient knew the partner was treated for the past infection. $\ddagger$ Estimates could not be obtained for white $v$ black because of insufficient cell sizes. $\S \mathrm{R}$, odds ratio; $\mathrm{Cl}$, confidence interval; adjusted for all other covariates in the table. 


\begin{tabular}{|c|c|c|c|}
\hline \multirow{2}{*}{$\begin{array}{l}\text { Table } 3 \\
\text { noted) }\end{array}$} & \multicolumn{3}{|c|}{ Significant correlates of knowledge of partner treatment ( $n=97$ unless otherwise } \\
\hline & $\begin{array}{l}\text { Knew partner was } \\
\text { treated }\end{array}$ & $\begin{array}{l}\text { Did not know partner } \\
\text { was treated }\end{array}$ & p Value \\
\hline \multicolumn{4}{|c|}{ Partnership status $(n=91)^{*}$} \\
\hline Same & $37(76 \%)$ & $12(24 \%)$ & 0.04 \\
\hline New & $23(55 \%)$ & $19(45 \%)$ & \\
\hline \multicolumn{4}{|c|}{ Age of patient (years) } \\
\hline $17-19$ & $51(73 \%)$ & $19(27 \%)$ & 0.02 \\
\hline $14-16$ & $13(48 \%)$ & $14(52 \%)$ & \\
\hline
\end{tabular}

restricted sample consisting of only those who were in the same relationship at both times $(49 / 91$ or $54 \%)$; results were in the same direction $(\mathrm{OR}=2.27,95 \% \mathrm{CI}$ : 0.33 to 15.63 , $\mathrm{p}=0.40$ ) but somewhat attenuated and not statistically significant. Adolescents who did not know if their partner was treated were equally likely to no longer be in the same relationship as those who reported their partner was not treated $(63 \% \vee 61 \%)$. A stratified analysis of knowledge of partner treatment by partnership status (same/new) at the time of STI testing indicated that those who did not know their partner was treated and were not in the same partnership had the highest probability of a current infection $(7 / 19=37 \%)$, whereas those who were in the same partnership and did know their partner was treated for the past infection had the lowest probability of current infection $(3 / 37=8 \%)(p=0.07)$.

Significant correlates of knowledge of partner treatment are presented in table 3. Seventy six per cent of those in the same partnership knew their partner was treated compared to $55 \%$ of those in new partnerships $(p=0.04)$, and $73 \%$ of older adolescents knew their partner was treated compared to $48 \%$ of younger adolescents $(p=0.02)$. None of the other tested demographic or sexual history covariates were significantly associated with knowledge of partner treatment.

\section{DISCUSSION}

The majority (95\%) of adolescents who were previously diagnosed with chlamydia or gonorrhoea reported that their healthcare provider recommended partner testing; however, a smaller proportion (66\%) reported knowing that their partner was treated. This estimate is comparable to the proportions found in other studies: 55\% of private sector patients in Seattle-King County, Washington, USA, reported knowing that all of their partners had been treated, ${ }^{20}$ and $47 \%$ of adolescent females in a multicentred US based study reported being "sure" that her partner had taken all his medication. ${ }^{5}$ The high frequency with which providers discuss the need for partner treatment with their patients observed in this study and in others, ${ }^{19}{ }^{20}$ but the lower frequencies at which adolescent females ensure that their partners are treated suggest greater efforts are needed to involve the patients directly in the treatment of their partners.

Not knowing that a partner was treated for past STI was independently associated with current infection $(\mathrm{OR}=4.5$, 95\% CI: 1.4 to 14.3). As also indicated in other studies ${ }^{4}$ current infections from the same (untreated) partner were likely in many cases, as many of the adolescents (54\%) reported being in the same partnership at the time of both infections. Furthermore, those who were not in the same relationship at the time of STI testing could have been reinfected from the previous, untreated partner before the end of that relationship. The lowest probability of current infection was found among those who were in the same partnership and knew the partner had been treated; this may reflect communicative and stable relationships. The highest probability of current infection was found among those who did not know their partner was treated and were not in the same partnership; these infections could have resulted from the previously untreated partner or from a new partner. In any case, not knowing that the previous partner was treated has important public health implications for the further spread of these infections to other sex partners in the community.

For young women who may have difficulty ensuring effective partner management on their own, an offer of assistance from a healthcare provider is likely to be an important step in the process. A number of partner management strategies are available to providers, including provider referral, health department referral, or patient delivered therapy as described in the introduction. Whether patients refer partners or deliver medications themselves, or cooperate with healthcare workers in more structured methods of provider referral, they ultimately have the critical role in the treatment of their partners because they must be willing to address the issue. How to best reach a partner will depend on the nature of the partnership; therefore, clinicians should work closely with their patients to determine which partner treatment strategy to use. Clearly, providers must emphasise not only partner referral, but also follow up on the part of the patient to ensure that the partner was in fact treated.

In this study we identified two potential barriers to knowing that partners were treated for the past infection. Participants ages 14-16 were significantly less likely to know whether their partner was treated compared to those who were aged 17-19. Therefore, clinicians should consider age of their patients when counselling them about treatment options for their partners. Younger adolescents may lack communication skills or be in relationships with unequal power differentials that make it difficult to raise questions about infidelities. A second potential barrier was being in a new sex partnership. The STI diagnosis may have resulted in the break up of the partnership. Alternatively, this could also reflect more casual partnerships in which there is little communication in general and about sensitive issues in particular. In both situations, it appears that the necessary communication after an STI diagnosis is not occurring. Whether the current relationship continues or not, the need for partner treatment must be emphasised during clinic visits with providers. In relationships that are likely to end, provider referral or field delivered therapy may be effective means of achieving partner treatment, as this does not require direct communication between patient and partner. In relationships that are likely to continue, providers may encourage a combined visit for the patient and her partner in which counselling is provided to both members of the partnership.

This study had several limitations. Firstly, without knowing the infection status of the partners, we are unable to 


\section{Key messages}

- In a sample of young women, not knowing that a sex partner was treated for a past bacterial STI was independently associated with having a current STI

- Clinicians should work closely with their patients to develop an effective partner management strategy, including offering assistance with notification or referral and encouraging partners' entry into care

- Ultimately, patients themselves have a critical role in ensuring partner treatment, and they should be encouraged to follow up directly with their partners regarding treatment

know for certain if the current infection was indeed from the same, previously untreated partner. However, after restricting the sample to only those in the same partnerships, we still observed the same trend. Furthermore, even among those in new partnerships it is possible that the current infection may have been acquired from the previous untreated partner before the formation of the new partnership. A second limitation is that we had limited data on the partnerships at the time of the self reported diagnoses, which precluded our ability to examine potentially important partnership level predictors of knowledge of partner treatment. Thirdly, our cross sectional design necessitated a reliance on self report for past information, though this is unlikely to have introduced bias because respondents were not aware of their current infection status at the time of the interview. Furthermore, self report of past diagnoses was comparable to diagnoses recorded in medical charts and reported to the state health department, indicating relatively adequate recall of this important variable. The statistical significance of our relation of interest indicates that small sample size was not a limitation of this study. This study also had several strengths, including a focus on a high risk group of adolescent women ${ }^{22-24}$ and the use of laboratory confirmed STI testing for current infections.

Enhanced efforts by both clinicians and patients are needed to ensure partner treatment for reducing the further spread of STI. Clinicians should encourage entry of partners into care for STI treatment and offer assistance when needed. Other partner treatment strategies such as patient delivered therapy may be useful in some circumstances. Interventions to increase partner treatment among this vulnerable population should be specifically tailored to address unique issues for young women, including the potential for difficulty communicating in relationships. Ultimately, increasing the frequency with which sex partners receive effective STI treatment will reduce the burden of these infections in young women, their partners, and the community.

\section{ACKNOWLEDGEMENTS}

This research was funded by the National Institute of Mental Health grant \#P01 MH/DA56826 and training grant \#T32 MH20031.

\section{CONTRIBUTORS}

LMN conceptualised the research question, conducted data management and analyses, and wrote the manuscript; JRI designed the study, oversaw its implementation, and provided comments on the analysis and manuscript; KZ provided comments on the analysis and manuscript; TSK assisted with data management and analyses, and provided comments on the analysis and manuscript; SM provided comments on the analysis and manuscript; JBL assisted with study design and implementation, and provided comments on the analysis and manuscript; KAE designed the study, oversaw its implementation, and provided comments on the analysis and manuscript.

\section{Authors' affiliations}

L M Niccolai, J R Ickovics, T S Kershaw, S Milan, J B Lewis, Yale University School of Medicine, Department of Epidemiology and Public Health, Center for Interdisciplinary Research on AIDS, New Haven, CT, USA

K Zeller, Yale University School of Medicine, Robert Wood Johnson Clinical Scholars Program, New Haven, CT, USA

K A Ethier, Centers for Disease Control and Prevention, Division of STD Prevention, Behavioral Intervention Research Branch, New Haven, CT, USA

Competing interests: none declared.

The Human Investigations Committees at Yale University School of Medicine (New Haven, CT, USA), and at all participating clinics approved access to and analyses of these data.

\section{REFERENCES}

1 Burstein GR, Gaydos CA, Diener-West M, et al. Incident Chlamydia trachomatis infections among inner-city adolescent females. JAMA 1998;280:521-6.

2 Fortenberry JD, Brizendine EJ, Katz BP, et al. Subsequent sexually transmitted infections among adolescent women with genital infection due to Chlamydia trachomatis, Neisseria gonorrhoeae, or Trichomonas vaginalis. Sex Transm Dis 1999;26:26-32.

3 DiClemente RJ, Wingood GM, Crosby RA, et al. Sexual risk behaviors associated with having older sex partners: a study of black adolescent females. Sex Transm Dis 2002;29:20-4.

4 Blythe MJ, Katz B, BE B, et al. Recurrent genitourinary chlamydial infections in sexually active adolescent females. J Pediatr 1992;121:487-93.

5 Kissinger $\mathbf{P}$, Clayton J, O'Brien $M$, et al. Older partners not associated with recurrence among female teenagers infected with Chlamydia trachomatis. Sex Transm Dis 2002;29:144-9.

6 Whittington WLH, Kent $C$, Kissinger $P$, et al. Determinants of persistent and recurrent Chlamydia trachomatis infection in young women: results of a multicenter cohort study. Sex Transm Dis 2001;28:117-23.

7 Orr D, Johnston K, Brizendine E, et al. Subsequent sexually transmitted infection in urban adolescents and young adults. Arch Pediatr Adolesc Med 2001; 155:947-53.

8 Oh KM, Cloud G, Fleenor M, et al. Risk for gonococcal and chlamydia cervicitis in adolescent females: incidence and recurrence in a prospective cohort study. J Adolesc Health 1996; 18:270-5.

9 Holmes KK, Sparling PF, Mardh P-A, et al. Sexually transmitted diseases. New York: McGraw-Hill, 1999.

10 Hillis S, Nakashima A, Marchbanks P, et al. Risk factors for recurrent Chlamydia trachomatis infections in women. Am J Obstetr Gynecol 1994; 170:801-6.

11 Laga M, Manoka A, Kivuvu M, et al. Non-ulcerative sexually transmitted diseases as risk factors for HIV-1 transmission in women: results from a cohort study. AIDS 1993;7:95-102.

12 Wasserheit JN. Epidemiological synergy: interrelationships between human immunodeficiency virus infection and other sexually transmitted diseases. Sex Transm Dis 1992; 19:61-77.

13 Centers for Disease Control and Prevention. Sexually transmitted diseases treatment guidelines. MMWR 2002;51.

14 Schillinger JA, Kissinger $\mathrm{P}$, Calvet $\mathrm{H}$, et al. Patient-delivered partner treatment with azithromycin to prevent repeated Chlamydia trachomatis infection among women. Sex Transm Dis 2003;30:49-56

15 Kissinger P, Brown R, Reed K, et al. Effectiveness of patient delivered partner medication for preventing recurrent Chlamydia trachomatis. Sex Transm Infect 1998;74:331-3.

16 Klausner JD, Chaw JK. Patient-delivered therapy for chlamydia: putting research into practice. Sex Transm Dis 2003;30:509-11.

17 Steiner KC, Davila V, Kent CK, et al. Field-delivered therapy increases treatment for chlamydia and gonorrhea. Am J Public Health 2003;93:882-4.

18 Golden MR, Whittington W, Handsfield $\mathrm{H}$, et al. Partner management for gonococcal and chlamydia infection: expansion of public health services to the private sector and expedited sex partner treatment through a partnership with commercial pharmacies. Sex Transm Dis 2001;28:658-65.

19 St Lawrence JS, Montano DE, Kasprzyk D, et al. STD screening, testing, case reporting, and clinical and partner notification practices: a national survey of US physicians. Am J Public Health 2002;92:1784-8.

20 Golden MR, Whittington WLH, Gorbach PM, et al. Partner notification for chlamydial infections among private sector clinicians in Seattle-King County: a clinician and patient survey. Sex Transm Dis 1999;26:543-7.

21 Golden MR, Hogben M, Handsfield HH, et al. Partner notification for HIV and STD in the United States: low coverage for gonorrhea, chlamydial infection, and HIV. Sex Transm Dis 2003;30:490-6.

22 Centers for Disease Control and Prevention. Sexually transmitted disease surveillance, 2001. Atlanta, GA: Department of Health and Human Services, 2002.

23 Niccolai LM, Ethier KA, Kershaw TS, et al. Pregnant adolescents at risk: sexual behaviors and STD prevalence. Am J Obstet Gynecol 2003;188:63-79.

24 Ickovics JR, Niccolai LM, Kershaw TS, et al. High post-partum rates of STDs among teens: Pregnancy as a window of opportunity for prevention. Sex Transm Infect 2003;79:469-73. 